\title{
Understanding the Role of RbgA in the Assembly of the 50S Ribosomal Subunit.
}

Joaquin Ortega ${ }^{1}$, Amal Seffouh ${ }^{1}$, Nikhil Jain ${ }^{2}$, Dushyant Jahagirdar ${ }^{1}$, Kaustuv Basu ${ }^{1}$, Aida Razi ${ }^{1}$, Xiaodan $\mathrm{Ni}^{3}$, Alba Guarne ${ }^{1}$ and Robert Britton ${ }^{2}$

${ }^{1}$ McGill University, Montreal, Quebec, Canada, ${ }^{2}$ Baylor College of Medicine, Houston, Texas, United States, ${ }^{3}$ McMaster University, Hamilton, Ontario, Canada

The ribosome is the enzyme responsible for protein synthesis and the most complex macromolecule existing in bacterial cells. In Escherichia coli, the ribosome is made from more than 50 different components organized into the small (30S) and large (50S) ribosomal subunits. In spite of its complexity, each bacterial cell assembles more than 20,000 ribosomes in less than 30 minutes. Cells are able to maintain this assembly rate because a number of assembly factors make the process extremely efficient. Many of these assembly factors are GTPases that are essential for growth. RbgA is one of these GTPases and is required for the assembly of the 50S subunit in most bacteria. Homologs of this protein are also implicated in the assembly of the large subunit of the mitochondrial and eukaryotic ribosome. We will present the cryo-electron microscopy structure of RbgA bound to a Bacillus subtilis 50S subunit assembly intermediate (45SRbgA particle) that accumulates in cells upon RbgA depletion. Binding of RbgA at the $\mathrm{P}$ site of the immature particle stabilizes functionally important rRNA helices in the A and P-sites, prior to the completion of the maturation process of the subunit. The structure also reveals the location of the highly conserved $\mathrm{N}$-terminal end of RbgA containing the catalytic residue Histidine 9 . The derived model supports a mechanism of GTP hydrolysis, and it shows that upon interaction of RbgA with the 45SRbgA particle, Histidine 9 positions itself near the nucleotide potentially acting as the catalytic residue with minimal rearrangements. In this process, helix 92 of the $23 \mathrm{~S}$ rRNA of the maturing ribosomal particle plays a key role by stabilizing the $\mathrm{N}$-terminal of RbgA and directly positioning the catalytic residue. This shows that the 23S rRNA functions in RbgA GTPase activation similarly to other TRAFAC-GTPases such as EF-Tu. This structure represents the first visualization of the conformational changes induced by an assembly factor in a bacterial subunit intermediate.

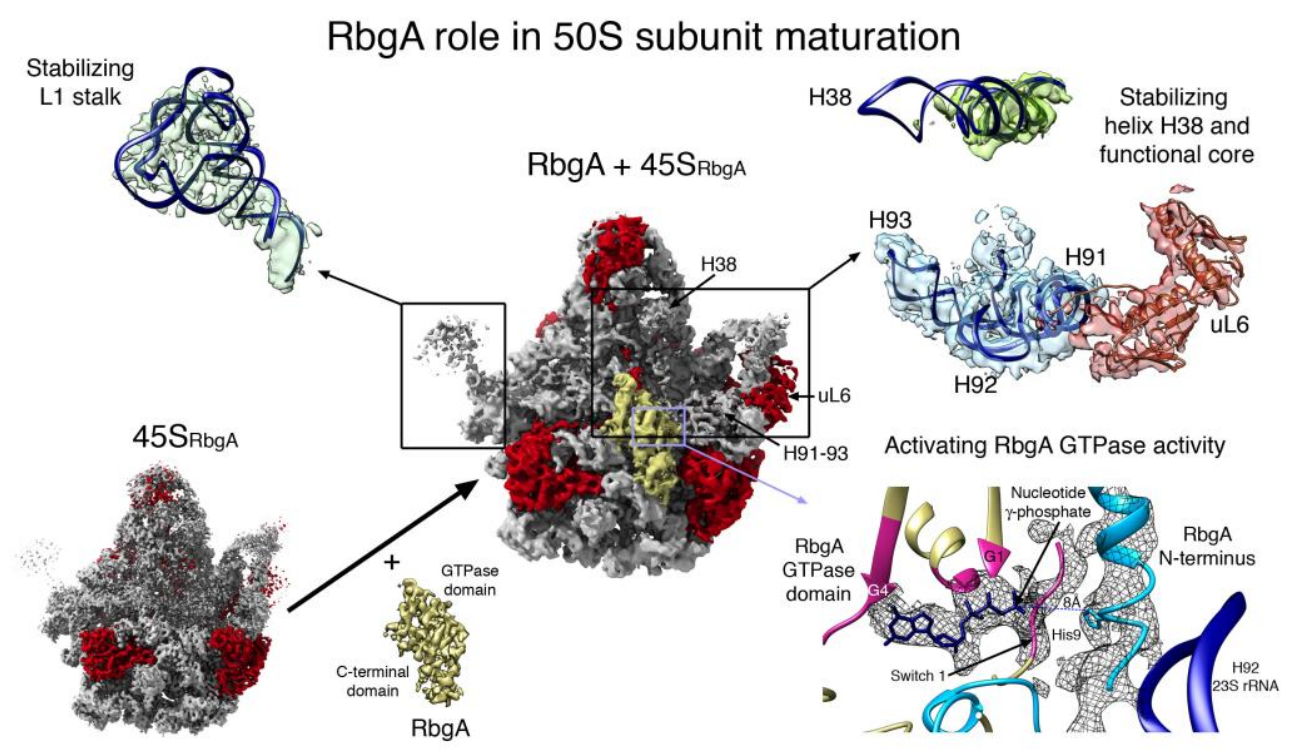

Figure 1. RbgA is an essential GTPase required for the assembly of the 50S subunit in most bacteria. Binding of $\mathrm{RbgA}$ at the $\mathrm{P}$ site of the 45SRbgA immature particle stabilizes helix 38 and other functionally 
important rRNA helices in the A and P-sites. The interaction of RbgA with the maturing ribosomal subunit also positions the catalytic residue His9 in the vicinity of the g-phosphate of the guanidine nucleotide suggesting a model for the mechanism of GTP hydrolysis.

\section{References}

Seffouh, A., Jain, N., Jahagirdar, D., Basu, K., Razi, A., Ni, X., Guarne, A., Britton, R.A., and Ortega, J. (2019). Structural consequences of the interaction of RbgA with a 50 S ribosomal subunit assembly intermediate. Nucleic Acids Res 47, 10414-10425. 